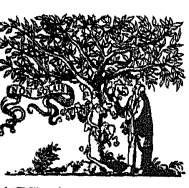

\title{
Projected dynamical systems in a complementarity formalism
}

\author{
W.P.M.H. Heemels ${ }^{\mathrm{a}, \mathrm{b}}$,, J.M. Schumacher ${ }^{\mathrm{b}, \mathrm{c}}$, S. Weiland ${ }^{\mathrm{a}}$ \\ a Department of Electrical Engineering, Eindhoven University of Technology, P.O. Box 513, \\ 5600 MB Eindhoven, The Netherlands \\ ${ }^{b}$ Department of Economics, Tilburg University, P.O. Box 90153, 5000 LE Tilburg, The Netherlands \\ ${ }^{\mathrm{c}}$ CWI, P.O. Box 94079, 1090 GB Amsterdam. The Netherlands
}

Received 1 April 1999; received in revised form 1 November 1999

\begin{abstract}
Projected dynamical systems have been introduced by Dupuis and Nagurney as dynamic extensions of variational inequalities. In the systems and control literature, complementarity systems have been studied as input/output dynamical systems whose inputs and outputs are connected through complementarity conditions. We show here that, under mild conditions, projected dynamical systems can be written as complementarity systems. (C) 2000 Elsevier Science B.V. All rights reserved.
\end{abstract}

Keywords: Variational inequalities; Complementarity; Discontinuous dynamical systems; Systems theory; Optimization

\section{Introduction}

In this paper, we connect two classes of discontinuous dynamical systems. One is the class of projected dynamical systems introduced by Dupuis and Nagurney [6] and further developed by Nagurney and Zhang [18]. These systems are described by differential equations of the form

$\dot{x}(t)=\Pi_{K}(x(t),-F(x(t)))$,

\footnotetext{
* Correspondence address. Department of Electrical Engineering, Eindhoven University of Technology, Bldg. E-hoog, Room 4.26, P.O. Box 513, 5600 MB Eindhoven, Netherlands. Fax: $+31-40-243-4582$.

E-mail addresses: w.p.m.h.heemels@tue.nl (W.P.M.H. Heemels), j.m.schumacher@cwi.nl (J.M. Schumacher), s.weiland @tue.nl (S. Weiland).
}

where $F$ is a vector field, $K$ is a closed convex set, and $\Pi_{K}$ is a projection operator that prevents the solution from moving outside the constraint set $K$ (cf. Section 2 for a precise definition). These systems are used for studying the behaviour of oligopolistic markets, urban transportation networks, traffic networks, international trade, and agricultural and energy markets (spatial price equilibria). Their stationary points can be characterized by means of variational inequalities; one may therefore say that projected dynamical systems provide a dynamic extension of variational inequalities.

We shall compare projected dynamical systems with complementarity systems, which may be considered as dynamical extensions of complementarity problems (cf. Section 3). Although particular forms of complementarity systems have been used for a long time in the context of specific applications such 
as electrical networks with ideal diodes (see, e.g. [2]) and mechanical objects subject to unilateral constraints [15], the idea of coupling complementarity conditions to a general input/output dynamical system has first been proposed in 1996 by van der Schaft and Schumacher [24]. Within the general class of dynamical systems that is obtained in this way, there is a natural subdivision corresponding to classifications that are used for input/output dynamical systems. In this paper we shall have occasion to use in particular the so-called gradient-type complementarity systems. To our knowledge this specific class of dynamical systems has not been introduced before in the literature. We note, though, that the class of nonsmooth dynamical systems recently studied by Seeger [27] contains systems that may be considered as reversed-time versions of gradient-type complementarity systems with linear dynamics.

Complementarity systems are nonsmooth dynamical systems; they switch between several dynamical regimes and may show impulsive motions resulting in discontinuities of some system variables. Since complementarity systems are subjected to both continuous dynamics and discrete switching, one may also consider them as a subclass of hybrid dynamical systems $[1,20,26]$. Because of the nonsmoothness of trajectories, the formulation of a solution concept for complementarity systems is nontrivial (see $[9,13,24,25]$ ). Questions of (local) existence and uniqueness of solutions have been studied under various assumptions in $[10,12-14,24,25]$. Analysis of numerical simulation methods based on time-stepping can be found in $[3,9]$.

The study of complementarity systems is motivated by a broad range of applications (see [11] for an overview): electrical networks with diodes, mechanical systems subject to unilateral constraints or Coulomb friction, control systems with relays, saturation characteristics or deadzones, variable structure systems, dynamical systems with static piecewise linear relations, hydraulic systems with one-way valves and optimal control problems with state or control constraints.

It is well known that variational inequalities and complementarity problems are closely related (see, for instance, Harker and Pang [8]). Therefore, it is reasonable to expect that projected dynamical systems and complementarity systems are also related. In this paper we show that there is indeed a natural relationship. Specializing to the stationary points, we obtain as a corollary the classical result which states that, under mild conditions, variational inequalities may be rewritten as mixed nonlinear complementarity problems [8, Proposition 2.2]. Moreover, we obtain a proof of existence and uniqueness of solutions of projected dynamical systems that is independent of the original proof by Dupuis and Nagurney [6] and in particular does not use the Skorokhod problem (see [28]). Complementarity systems have already been used extensively in the engineering literature (see, for instance, $[15,19,25]$ ) and the establishment of a relation between the domains of projected dynamical systems and of complementarity systems makes it possible to compare and transfer analytic and computational techniques between the two. It also follows that the interpretation of projected dynamical systems as tatonnement or adjustment processes carries over to a class of complementarity systems.

The following notational conventions and terminology will be used. If $k$ is a positive integer, $\bar{k}$ denotes the set $\{1, \ldots, k\}$. For an index set $I \subseteq \bar{k}$, we denote its complement with respect to $\bar{k}$ by $I^{\mathrm{c}}:=\{i \in \bar{k} \mid i \notin I\}$. The cardinality of a set $I$ will be denoted by $|I|$. A vector $u \in \mathbb{R}^{k}$ is said to be nonnegative (nonpositive) if $u_{i} \geqslant 0\left(u_{i} \leqslant 0\right)$ for all $i \in \bar{k}$, and in this case we write $u \geqslant 0(u \leqslant 0)$. Given a matrix $M \in \mathbb{R}^{k \times l}$ and subsets $I \subseteq \bar{k}$ and $J \subseteq \bar{l}$, we denote the submatrix $\left(M_{i j}\right)_{i \in l, j \in J}$ by $M_{I J}$. In case $I=\bar{k}$ we write $M_{\bullet J}$ rather than $M_{\bar{k} J}$, and similarly if $J=\bar{l}$ we use $M_{l}$. The transpose of a matrix $M$ is denoted by $M^{\mathrm{T}}$. In the Euclidean space $\mathbb{R}^{k}$ the standard inner product is denoted by $\langle\cdot, \cdot\rangle$ and for $u, v \in \mathbb{R}^{k}$ we write $u \perp v$ if $\langle u, v\rangle=u^{\mathrm{T}} v=0$. We denote the restriction of a function $f:[0, T] \rightarrow \mathbb{R}$ to an inter$\operatorname{val}(a, b) \subseteq[0, T]$ by $\left.f\right|_{(a, b)}$. A function $f: \mathbb{R}^{n} \rightarrow \mathbb{R}^{p}$ will be said to be real-analytic and convex if its component functions $f_{i}: \mathbb{R}^{n} \rightarrow \mathbb{R}$ are real-analytic and convex.

\section{Projected dynamical systems}

In this section we recall the definition of projected dynamical systems (PDS) $[6,18]$. The defining ingredients are a closed convex set $K$, which usually corresponds to the constraint set of a particular application, and a vector field $F$ whose domain contains $K$. The projected dynamics is described by the equation 
$\dot{x}(t)=-F(x(t))$ on the interior of $K$, but on the boundary a modification is applied to prevent the solution from leaving the constraint set.

To be more precise, let a closed and convex set $K \subseteq \mathbb{R}^{n}$ be given. The cone of inward normals at $x \in$ $K$ is defined by

$$
n(x)=\{\gamma \mid\langle\gamma, x-k\rangle \leqslant 0 \text { for all } k \in K\} \text {. }
$$

Note that $n(x)=\{0\}$, when $x$ is contained in the interior of $K$. Given $x \in K$ and $v \in \mathbb{R}^{n}$, define the projection of the vector $v$ at $x$ with respect to $K$ by

$\Pi_{K}(x, v)=v-\left\langle v, n^{*}(x)\right\rangle n^{*}(x)$,

where

$n^{*}(x) \in \arg \max _{n \in n(x),\|n\| \leqslant 1}\langle v,-n\rangle$.

Note that $\Pi_{K}(x, v)$ is well-defined even though $n^{*}(x)$ may not be uniquely specified by (3b). The projected dynamical system $\operatorname{PDS}(F, K)$ corresponding to a closed convex set $K$ and a vector field $F$ on $K$ is defined by

$\dot{x}(t)=\Pi_{K}(x(t),-F(x(t)))$.

The ordinary differential equation (4) has a discontinuous right-hand side and is therefore not covered by the standard theory of differential equations. The following notion of solution is proposed in [18].

Definition 2.1 (Nagurney and Zhang [18]). An absolutely continuous function $x:[0, T] \rightarrow K$ is a solution to $\operatorname{PDS}(F, K)$ on $[0, T]$ with initial state $x_{0} \in K$ if $x(0)=x_{0}$ and (4) holds almost everywhere in $[0, T]$.

Definition (3) of the projection operator $\Pi_{K}$ is convenient for the development below. An alternative definition is the following one. For $x \in K$ and $v \in \mathbb{R}^{n}$ define

$\Pi_{K}(x, v)=\lim _{\delta \rightarrow 0} \frac{P_{K}(x+\delta v)-x}{\delta}$,

where $P_{K}$ is the projection operator that assigns to each vector $x$ in $\mathbb{R}^{n}$ the vector in $K$ that is closest to $x$ in the Euclidean norm $\|\cdot\|$ (i.e. $P_{K} x=\arg \min _{k \in K}\|x-k\|$ ). It has been proven by Dupuis [5] that the formulations in (3) and (5) are equivalent when $K$ is convex and compact with nonempty interior. In [6] the same result is stated under the assumption that $K$ is a convex polyhedron (i.e. an intersection of finitely many closed half-spaces ).

\section{Complementarity systems}

A complementarity system may be specified (in "semi-explicit affine form", see [24]) by functions $f: \mathbb{R}^{n} \rightarrow \mathbb{R}^{n}, g_{i}: \mathbb{R}^{n} \rightarrow \mathbb{R}^{n}$ and $h: \mathbb{R}^{n} \rightarrow \mathbb{R}^{p}$. The defining equations for the complementarity system corresponding to $f, g_{i}$ and $h$ are

$\dot{x}(t)=f(x(t))+\sum_{i=1}^{p} g_{i}(x(t)) u_{i}(t)$,

$y(t)=h(x(t))$

$0 \leqslant y(t) \perp u(t) \geqslant 0$.

Relation (6c) implies that for all $i$ at least one of the equalities $u_{i}(t)=0$ and $y_{i}(t)=0$ must be satisfied. Hence, for all times $t$ there exists an index set $J$ such that $u_{i}(t)=0, i \notin J$ and $y_{i}(t)=0, i \in J$. In the engineering literature this index set is sometimes called the active index set, mode or discrete state of the system at time $t$. The mode may change during the time evolution of the system. The times at which this happens are called event times.

In general, a complementarity system may not have a continuous solution, even when the defining functions $f, g$ and $h$ are smooth, and so one needs to introduce larger function spaces to define solutions (cf. $[12,13,24,25])$. Although the solution concept below is not the most general one, it suffices for the purpose of the paper. We need the notion of right-isolated sets. A subset $\mathscr{E}$ of $\mathbb{R}$ is said to be right-isolated if for each $t \in \mathscr{E}$ there exists an $\varepsilon>0$ such that $(t, t+\varepsilon) \cap \mathscr{E}=\emptyset$.

Definition 3.1. A continuous function $x:[0, T] \rightarrow$ $\mathbb{R}^{n}$ is called a solution to (6) with initial state $x_{0}$ on the interval $[0, T]$, if $x(0)=x_{0}$ and there exist a right-isolated set $\mathscr{E} \subset[0, T]$ and two functions $u$ : $[0, T] \rightarrow \mathbb{R}^{p}, y:[0, T] \rightarrow \mathbb{R}^{p}$ such that for any interval $(a, b) \subseteq[0, T]$ with $(a, b) \cap \mathscr{E}=\emptyset$ the following conditions hold:

1. the restriction $\left.(u, x, y)\right|_{(a, b)}$ is real-analytic and satisfies (6a) and (6b) for all $t \in(a, b)$;

2. there exists an index set $J \subseteq \bar{p}$ such that $u_{J^{c}}(t)=0$, $y_{J}(t)=0, u_{J}(t) \geqslant 0$ and $y_{J^{c}}(t) \geqslant 0$ for all $t \in(a, b)$.

This definition allows solutions that exhibit accumulations of event times ("Zeno solutions"). Since $\mathscr{E}$ 
is right-isolated, such accumulations only take place forward in time. Note that a similar restriction is not present in Definition 2.1.

By considering several types of dynamics in (6a) and (6b), one may define several classes of complementarity systems such as linear complementarity systems $[13,24]$ and Hamiltonian complementarity systems [24]. For the purpose of this paper we shall be particularly interested in gradient-type complementarity systems; these systems are related to the gradient systems that have been studied by van der Schaft [23]. To specify a gradient-type complementarity system, take functions $F: \mathbb{R}^{n} \rightarrow \mathbb{R}^{n}$ and $h: \mathbb{R}^{n} \rightarrow \mathbb{R}^{p}$. Let the gradients of the component functions $h_{i}(x)$ of $h(x)$ be denoted by $\nabla h_{i}(x)$ (taken to be row-vectors) and let $H(x)$ denote the matrix whose $i$ th row is equal to $\nabla h_{i}(x)$ (i.e. the Jacobian matrix of $h$ at $x$ ). The gradient-type complementarity system $\operatorname{GTCS}(F, h)$ is given by the equations

$$
\begin{aligned}
& \dot{x}(t)=-F(x(t))+\sum_{i=1}^{p}\left[\nabla h_{i}(x(t))\right]^{\mathrm{T}} u_{i}(t), \\
& y(t)=h(x(t)), \\
& 0 \leqslant y(t) \perp u(t) \geqslant 0,
\end{aligned}
$$

which is a special case of (6). Eq. (7a) can compactly be written in terms of the Jacobian $H$ of $h$ as

$$
\dot{x}(t)=-F(x(t))+[H(x(t))]^{\mathrm{T}} u(t) .
$$

The above definition makes implicit use of the standard inner product of $\mathbb{R}^{n}$, but it would also be possible to use a coordinate-free treatment as in [23]. There is a closer analogy with the gradient systems studied by van der Schaft when in (7) the function $F$ is defined as the gradient of some potential function. In that case (7) is referred to as a gradient complementarity system.

\section{Projected dynamical systems as complementarity systems}

In this section we consider projected dynamical systems specified by a vector field $F$ and a convex set $K$, and we provide conditions under which these systems can be rewritten as gradient-type complementarity systems. It will be assumed that the convex set $K$ can be represented by means of finitely many inequalities.
Assumption 4.1. The set $K$ allows a representation in the form

$K=\left\{x \in \mathbb{R}^{n} \mid h(x) \geqslant 0\right\}$,

where $h: \mathbb{R}^{n} \rightarrow \mathbb{R}^{p}$ is real-analytic and convex.

If $h$ represents $K$ as in (9), we define for $x \in K$ the active index set $I(x)$ as

$I(x):=\left\{i \in \bar{p} \mid h_{i}(x)=0\right\}$.

To prevent technical complications that would obscure the main line of reasoning, we shall use the following constraint qualification in conjunction with Assumption 4.1.

Assumption 4.2. For $h$ as in (9) and $H$ the Jacobian of $h$, the matrix $H_{I(x) \bullet}(x)$ has full row rank for all $x \in K$.

Concerning the vector field $F$, we shall use the following assumptions.

Assumption 4.3. The vector field $F$ is real-analytic.

Assumption 4.4. There exists a constant $B \in \mathbb{R}$ such that $F$ satisfies the linear growth condition

$\|F(x)\| \leqslant B(1+\|x\|)$ for all $x \in K$.

Assumption 4.5. There exists a constant $C \in \mathbb{R}$ such that

$\langle-F(x)+F(y), x-y\rangle \leqslant C\|x-y\|^{2}$

for all $x, y \in K$.

Remark 4.6. Assumption 4.1 implies that $K$ is convex and closed. A characterization of $K$ as in (9) is possible in all applications of projected dynamical systems mentioned in [18]. In [6] it is even assumed that $K$ is a convex polyhedron. Assumptions 4.4 and 4.5 are used in [18] to prove existence and uniqueness of solutions to the projected dynamical system specified by $F$ and $K$.

The following theorem is the main result of this paper. The theorem will be proved in the next section.

Theorem 4.7. Let a set $K \subseteq \mathbb{R}^{n}$, a vector field $F: \mathbb{R}^{n} \rightarrow \mathbb{R}^{n}$ and a function $h: \mathbb{R}^{n} \rightarrow \mathbb{R}^{p}$ be given 
such that Assumptions 4.1-4.5 are satisfied. For all initial states $x_{0} \in K$, both the projected dynamical system $\operatorname{PDS}(F, K)$ and the gradient-type complementarity system $\operatorname{GTCS}(F, h)$ have a unique solution defined on $[0, \infty)$. Moreover, these solutions coincide.

Remark 4.8. It will follow from the proof given below that without Assumption 4.4 the theorem still holds, except that the solutions are not guaranteed to exist on $[0, \infty)$. To be specific, suppose that $\left[0, T_{1}\right)$ is the maximal interval on which a solution can be defined for $\operatorname{PDS}(F, K)$. Similarly, let $\left[0, T_{2}\right)$ be the maximal interval for which $\operatorname{GTCS}(F, h)$ admits a solution. Then $T:=T_{1}=T_{2}>0$, both solutions are unique on $[0, T)$, and the solutions are equal to each other.

Remark 4.9. The constraint qualification Assumption 4.2 is introduced here for simplicity. In the literature on complementarity systems, weaker assumptions have been used. Specifically, Lötstedt [15] uses the condition that the Jacobian matrix $H(x)$ should have locally constant row rank to prove the existence and uniqueness of solutions to equations representing unilaterally constrained mechanical systems.

Remark 4.10. Theorem 4.7 provides some additional information about the solutions to $\operatorname{PDS}(F, K)$. Under the assumptions of the theorem, solutions to projected dynamical system are real-analytic on the open intervals belonging to a set of the form $[0, \infty) \backslash \mathscr{E}$. Moreover, the exceptional set (the set of event times) $\mathscr{E}$ is a right-isolated set.

Remark 4.11. It follows in particular that, under the conditions of Theorem 4.7 , the stationary points of the projected dynamical system $\operatorname{PDS}(F, K)$ coincide with those of the gradient-type complementarity system $\operatorname{GTCS}(F, h)$. When $K$ is a convex polyhedron, the stationary points $\bar{x}$ of $\operatorname{PDS}(F, K)$ are given by the variational inequality [18, Lemma 1$]$

$\langle F(\bar{x}), x-\bar{x}\rangle \geqslant 0 \quad \forall x \in K$.

The stationary points $\bar{x}$ of $\operatorname{GTCS}(F, h)$ are given by the mixed nonlinear complementarity problem

$0=-F(\bar{x})+\sum_{i=1}^{p}\left[\nabla h_{i}(\bar{x})\right]^{\mathrm{T}} u_{i}$ $y=h(\bar{x})$,

$0 \leqslant y \perp u \geqslant 0$.

In this way, we recover the well-known result (see for instance [8, Proposition 2.2]) that, under a suitable constraint qualification, variational inequalities may be rewritten as mixed nonlinear complementarity problems.

\section{Proof of the main result}

We start with a characterization of the projection $\Pi_{K}$ in terms of a minimization problem. The proof will be given below on the basis of a duality argument.

Theorem 5.1. Let $K \subset \mathbb{R}^{n}$ be of the form (9) for a real-analytic and convex function $h: \mathbb{R}^{n} \rightarrow \mathbb{R}^{p}$. For all $x \in K$ and $v \in \mathbb{R}^{n}$, we have

$\Pi_{K}(x, v)=\arg \min _{w \in W^{\prime}(x)}\|w-v\|$,

where $W(x)$ is the "cone of admissible velocities" given by

$W(x)=\left\{w \in \mathbb{R}^{\prime \prime} \mid \nabla h_{i}(x) w \geqslant 0\right.$ for all $\left.i \in I(x)\right\}$

with $I(x)$ specified by (10).

The duality result that we will use to prove Theorem 5.1 is stated in Proposition 5.2. The notation $C^{0}$ is used to denote the polar cone (see e.g. [21, p. 121]) of a set $C \subseteq \mathbb{R}^{n}$ :

$C^{0}=\left\{x \in \mathbb{R}^{n} \mid\langle x, y\rangle \leqslant 0\right.$ for all $\left.y \in C\right\}$.

Proposition 5.2. Let $W \subseteq \mathbb{R}^{n}$ be a closed convex cone with nonempty interior and let $v \in \mathbb{R}^{n}$ be given. Define $w^{*}$ by

$w^{*}=\arg \min _{w^{\prime} \in W}\|w-v\|$

and let $z^{*}$ be such that

$z^{*} \in \arg \max _{z \in W^{0},\|z\| \leqslant 1}\langle v, z\rangle$.

Then

$w^{*}=v-\left\langle v, z^{*}\right\rangle z^{*}$. 
Proof. We apply the Fenchel duality theorem [16, p. $201]$ to the convex function $f(w):=\|w-v\|$ defined on $C:=\mathbb{R}^{n}$ and the concave function $g(w):=0$ defined on $D:=W$. One easily computes (cf. for instance [21, Section 12]) that the conjugate sets of $C$ and $D$ are $C^{*}=\left\{z \in \mathbb{R}^{n} \mid\|z\| \leqslant 1\right\}$ and $D^{*}=-W^{0}$, and that the conjugate functions of $f$ and $g$ are given by $f^{*}(z)=\langle v, z\rangle$ for $z \in C^{*}$ and $g^{*}(z)=0$ for $z \in D^{*}$. From the Fenchel duality theorem, we therefore have

$\min _{w \in W}\|w-v\|=\max _{z \in W^{0},\|z\| \leqslant 1}\langle v, z\rangle$.

Now, suppose first that $\min _{w \in W}\|w-v\|>0$; then $\left\|z^{*}\right\|=1$. In this case, there exists a real number $\alpha \geqslant 0$ such that $w^{*}-v=-\alpha z^{*}[16$, p. 136]. We have $-\alpha=$ $-\left\|\alpha z^{*}\right\|=-\left\|w^{*}-v\right\|=-\left\langle v, z^{*}\right\rangle$ by (21); this proves (20). Next, suppose that $\min _{w \in W}\|w-v\|=0$. Then $v \in$

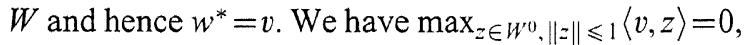
so that $\left\langle v, z^{*}\right\rangle=0$ and consequently Eq. (20) is also correct in this case.

Remark 5.3. The proof implies that $\left\langle w^{*}, z^{*}\right\rangle=0$. Together with the conditions $w^{*} \in W, z^{*} \in W^{0}$, and $v=w^{*}+\left\langle v, z^{*}\right\rangle z^{*}$, this shows that $\left\langle v, z^{*}\right\rangle z^{*}$ is actually the projection $P_{W^{0}} v$ of $v$ onto the cone $W^{0}[17$, p. 238].

Proof of Theorem 5.1. Fix an arbitrary $x \in K$. From [21, Corollaries 23.7.1 and 23.8.1] it follows that the cone of inward normals of $K$ at $x$, denoted by $n(x)$, and the cone of inward normals of $W(x)$ at 0 , denoted by $n_{W(x)}(0)$ satisfy

$$
\begin{aligned}
n(x)= & n_{W(x)}(0) \\
= & \left\{\gamma \in \mathbb{R}^{n} \mid \gamma=\sum_{i \in I(x)}\left[\nabla h_{i}(x)\right]^{\mathrm{T}} \lambda_{i}\right. \\
& \text { for certain } \left.\lambda_{i} \geqslant 0\right\} .
\end{aligned}
$$

By definition of the cone of inward normals and the polar cone (see (2) and (17)), $n_{W(x)}(0)$ is equal to $-W(x)^{0}$. Hence, $n(x)=-W(x)^{0}$. The claim of the theorem now follows by applying Proposition 5.2 to $W=W(x)$ and using that $W^{0}=-n(x)$.

Next, we establish a connection to a linear complementarity problem (LCP). See [4] for an extensive treatment of LCPs.
Theorem 5.4. Let a subset $K$ of $\mathbb{R}^{n}$ be of the form (9) for a real-analytic and convex function $h: \mathbb{R}^{n} \rightarrow$ $\mathbb{R}^{p}$. Fix $x \in K$. Let $H$ be the Jacobian matrix of $h$ at $x$, and let $I:=I(x)=\left\{i \mid h_{i}(x)=0\right\}$ be the active index set. Then we have

$\Pi_{K}(x, v)=v+H_{I \bullet}^{\mathrm{T}} u$

where the vector $u \in \mathbb{R}^{|I|}$ solves the $\mathrm{LCP}$

$0 \leqslant u \perp H_{l} \bullet+H_{l}\left[H_{I \bullet}\right]^{\mathrm{T}} u \geqslant 0$.

Proof. By Theorem 5.1, the vector $\Pi_{K}(x, v)$ is the projection of $v$ onto the cone $W(x)$ defined in (16). In terms of the notation introduced in the statement of the theorem, we have

$W(x)=\left\{w \in \mathbb{R}^{n} \mid H_{l} \bullet w \geqslant 0\right\}$.

The fact that the projection onto this cone can be found from (23) is well known; one may for instance use the Kuhn-Tucker conditions. An alternative approach is to use the result by Moreau [17] which states that in order to compute the projection of a vector $v$ in a Hilbert space on a closed cone $W$, it is enough to find $w$ and $w^{0}$ such that $v=w+w^{0}, w \in W, w^{0} \in W^{0}$, and $w \perp w^{0}$; the projection $P_{W} v$ is then given by $w$. In our case $W(x)$ is given by (24) so that the polar cone $W^{0}(x)$ can be written as (see e.g. the proof of Theorem 5.1)

$$
\begin{gathered}
W^{0}(x)=\left\{w^{0} \in \mathbb{R}^{n} \mid w^{0}=-\left[H_{l \bullet}\right]^{\mathrm{T}} u\right. \\
\text { for some } u \geqslant 0\} .
\end{gathered}
$$

Therefore, the three conditions of the LCP (23b) are exactly the conditions that ensure, by Moreau's theorem, that $\Pi_{K}(x, v)$ is given by (23). Note in particular that the condition $\left[H_{l \bullet}\right]^{\mathrm{T}} u \perp v+\left[H_{l}\right]^{\mathrm{T}} u$ is equivalent to $u \perp H_{l} \bullet v+H_{l} \bullet\left[H_{l} \bullet\right]^{\mathrm{T}} u$.

The discussion so far may be summarized as follows.

Corollary 5.5. A function $x:[0, T] \rightarrow \mathbb{R}^{n}$ is a solution to the projected dynamical system (4) if and only if there exists a locally integrable function $u$ : $[0, T] \rightarrow \mathbb{R}^{p}$ such that, with $I(x)$ the active index set as in (10) and $H(x)$ the Jacobian matrix of $h$ at $x \in$ $K$, one has for almost all $t \in[0, T]$ :

$\dot{x}(t)=-F(x(t))+\left[H_{I(x(t)) \bullet}(x(t))\right]^{\mathrm{T}} u_{I(x(t))}(t)$, 


$$
\begin{aligned}
& u_{I(x(t))^{c}}(t)=0 \\
& \begin{aligned}
0 \leqslant & u_{I(x(t))}(t) \perp-H_{I(x(t)) \bullet}(x(t)) F(x(t)) \\
& \quad+H_{I(x(t)) \bullet}(x(t))\left[H_{I(x(t)) \bullet}(x(t))\right]^{\mathrm{T}} u_{I(x(t))}(t) \\
\geqslant & 0 .
\end{aligned}
\end{aligned}
$$

In the proof of the main theorem we shall use the following result, which can easily be derived from Theorem 3.2 in [25]. The quoted theorem gives a local existence and uniqueness result for complementarity systems of the form (6).

Theorem 5.6. Let real-analytic functions $F: \mathbb{R}^{n} \rightarrow$ $\mathbb{R}^{n}$ and $h: \mathbb{R}^{n} \rightarrow \mathbb{R}^{p}$ be given. Take $x_{0} \in \mathbb{R}^{n}$ such that $h\left(x_{0}\right) \geqslant 0$. If Assumption 4.2 is satisfied, then there exists an $\varepsilon>0$ such that $\operatorname{GTCS}(F, h)$ has a solution $x$ on $[0, \varepsilon)$ with initial condition $x_{0}$. Moreover, this solution is unique.

Proof. Define $I=I\left(x_{0}\right)$ as in (10) and apply Theorem 3.2 in [25] to the system $\operatorname{GTCS}\left(F, h_{l}\right)$, i.e. $\dot{x}(t)=$ $-F(x(t))+\left[H_{I} \bullet(x(t))\right]^{\mathrm{T}} u_{I}(t)$ and $0 \leqslant h_{I}(x(t)) \perp u_{I}(t)$ $\geqslant 0$ with $I=I\left(x_{0}\right)$. Since $h_{i}\left(x_{0}\right)>0$ for $i \notin I\left(x_{0}\right)$, it is clear that continuous solutions to $\operatorname{GTCS}\left(F, h_{l}\right)$ with initial state $x_{0}$ are solutions to $\operatorname{GTCS}(F, h)$ for sufficiently small $t$, and vice versa.

Note that $H_{I} \bullet\left(x_{0}\right)\left[H_{I} \bullet\left(x_{0}\right)\right]^{\mathrm{T}}$ is positive definite due to Assumption 4.2 and hence, is also a $P$-matrix (i.e. has only positive principal minors ) [4, Theorems 3.1.6 and 3.3.7]. Consequently, Theorem 3.2 in [25] applies to $\operatorname{GTCS}\left(F, h_{l}\right)$ and the result follows.

Now we are in a position to prove the main result of this paper.

Proof of Theorem 4.7. Take $x_{0} \in K$. According to Theorem 5.6 there exists a real-analytic triple $(u, x, y)$ that satisfies $(7)$ on $[0, \varepsilon)$. In particular, there exists an index set $J \subseteq \bar{p}$ such that $y_{J}(t)=0$ and $u_{J c}(t)=0$ for all $t \in[0, \varepsilon)$.

We now want to show that the trajectory $x$ that has been defined in this way on $[0, \varepsilon)$ is also a solution to $\operatorname{PDS}(F, K)$ on $[0, \varepsilon)$. It is immediately clear that $(26 \mathrm{a})$ is satisfied because it is just another way of writing (8). For $x \in K$, define $I(x)$ as in (10). From the fact that $y_{J}(t)=0$ on $[0, \varepsilon)$ it follows that $J \subseteq I(x(t))$ for $t \in$ $[0, \varepsilon)$. Therefore, $I(x(t))^{\mathrm{c}} \subseteq J^{\mathrm{c}}$ and so $u_{I(x(t))^{\mathrm{c}}}(t)=0$ for $t \in[0, \varepsilon)$. Hence, $(26 \mathrm{~b})$ is satisfied. It remains to show that $u_{I(x(t))}(t)$ satisfies the LCP $(26 \mathrm{c})$ on $(0,8)$. It is clear from (7c) that the inequality $u_{I(x(t))}(t) \geqslant 0$ is satisfied on $(0, \varepsilon)$. For $t \in(0, \varepsilon)$, we have

$$
\begin{aligned}
0=\dot{y}_{J}(t)= & -H_{J} \bullet(x(t)) F(x(t)) \\
& +H_{J \bullet}(x(t))\left[H_{J} \bullet(x(t))\right]^{\mathrm{T}} u_{J}(t) .
\end{aligned}
$$

Dropping all arguments now to lighten the notation, we have from $u_{J^{\mathrm{c}}}=0$ and $J \subseteq I$ that

$\left(H_{l \bullet}\left[H_{l} \bullet\right]^{\mathrm{T}} u_{l}\right)_{J}=H_{J \bullet}\left[H_{J}\right]^{\mathrm{T}} u_{J}$.

Since obviously $\left(H_{l} \cdot F\right)_{J}=H_{J} F$, it follows from (27) and from $u_{J^{c}}=0$ that the orthogonality condition in (26c) holds. The final inequality in $(26 c)$ follows by expressing $\dot{y}_{i}(t)$ similarly to $(27)$, and noting that $\dot{y}_{i}(t) \geqslant 0$ whenever $y_{i}(t)=0$ (i.e. whenever $i \in$ $I(x(t)))$, because otherwise the inequality $y_{i}(t) \geqslant 0$ on $[0, \varepsilon)$ would be violated.

If the $\operatorname{limit}_{\lim _{t}} x(t)=: x(\varepsilon)$ exists, the existence of a solution to (7) starting from $x(\varepsilon)$ on $\left[\varepsilon, \varepsilon+\varepsilon_{1}\right)$ for some $\varepsilon_{1}>0$ follows from Theorem 5.6. Hence, we have a solution $x$ to $(7)$ on $\left[0, \varepsilon+\varepsilon_{1}\right)$ in the sense of Definition 3.1. In the same way as above, it can be shown that $x$ is a solution of $\operatorname{PDS}(F, K)$ on $\left[0, \varepsilon+\varepsilon_{1}\right)$.

We now have to show that actually a solution to $\operatorname{GTCS}(F, h)$ can be constructed on all of $[0, \infty)$. In principle it might happen that the above construction only leads to a solution on some interval $[0, T)$ with $T<\infty$. To proceed by contradiction, assume that we are in such a situation. The following estimates hold for $0 \leqslant t \leqslant T$ :

$$
\begin{aligned}
\|x(t)\| & \leqslant\left\|x_{0}\right\|+\int_{0}^{t}\left\|\Pi_{K}(x(\tau),-F(x(\tau)))\right\| \mathrm{d} \tau \\
& \leqslant\left\|x_{0}\right\|+\int_{0}^{t}\|F(x(\tau))\| \mathrm{d} \tau \\
& \leqslant\left\|x_{0}\right\|+B T+B \int_{0}^{t}\|x(\tau)\| \mathrm{d} \tau .
\end{aligned}
$$

The second step follows easily from the definition of $\Pi_{K}$ (see [18, Eq. (2.19)] or use the orthogonality mentioned in Remark 5.3) and the third inequality is a consequence of (11). Using Gronwall's lemma we see from this that $x(\cdot)$ is bounded on $[0, T)$; say $\|x(t)\| \leqslant M$ for $t \in[0, T)$ for some constant $M>0$. It follows in particular that no "finite-escape time" can occur. Moreover, it follows that the solution $x$ is 
Lipschitz continuous and hence uniformly continuous on $[0, T)$. Indeed, for $0 \leqslant t<s<T$ we have

$$
\begin{aligned}
\|x(t)-x(s)\| & \leqslant \int_{t}^{s}\left\|\Pi_{K}(x(\tau),-F(x(\tau)))\right\| \mathrm{d} \tau \\
& \leqslant \int_{t}^{s}\|F(x(\tau))\| \mathrm{d} \tau \\
& \leqslant B \int_{t}^{s}(1+\|x(\tau)\|) \mathrm{d} \tau \\
& \leqslant B(1+M)(s-t) .
\end{aligned}
$$

By a standard result in analysis (see for instance [22, Excercise 4.13]) this implies that the limit $x(T):=\lim _{t \uparrow T} x(t)$ exists. Since by continuity arguments $h(x(T)) \geqslant 0$, continuation is possible beyond $T$ according to Theorem 5.6, and we have reached a contradiction. Therefore, it follows that there is a unique solution of the gradient-type complementarity system $\operatorname{GTCS}(F, h)$ on $[0, \infty)$ which is also a solution of the projected dynamical system $\operatorname{PDS}(F, K)$. The uniqueness of solutions to $\operatorname{PDS}(F, K)$ follows from Assumption 4.5 as in [18, p. 33].

Remark 5.7. The existence of solutions to $\operatorname{PDS}(F, K)$ on $[0, \infty)$ is shown in [28] by a method based on the Skorokhod Problem [28]. The proof above provides an alternative argument. In fact the proof shows that Assumptions 4.1-4.3 are sufficient for local existence of solutions to $\operatorname{PDS}(F, K)$. With the additional Assumption 4.4 , one can prove existence on $[0, \infty)$. The argument to prove uniqueness uses Assumption 4.5 and is essentially due to Filippov [7].

\section{Conclusions}

We have shown that, under mild conditions, projected dynamical systems can be rewritten as gradient-type complementarity systems. This result may be looked at as a dynamic version of the well-known fact that, under suitable conditions, variational inequalities may be rewritten as mixed nonlinear complementarity problems. The class of gradient-type complementarity systems is a subclass of the class of complementarity systems which has received a considerable amount of attention in the engineering and applied physics literature. The establishment of a connection between the domains of projected dynamical systems and complementarity systems facilitates the transfer of techniques from one domain to the other. As an interesting bonus, we have obtained a new, and in the authors' opinion more direct, proof for the existence of solutions to projected dynamical systems.

\section{References}

[1] P.J. Antsaklis, A. Nerode (Guest Eds.), Special issue on hybrid control systems, IEEE Trans. Automa. Control 43 (4) (1998).

[2] W.M.G. van Bokhoven, Piecewise Linear Modelling and Analysis, Kluwer, Deventer, The Netherlands, 1981.

[3] M.K. Çamlibel, W.P.M.H. Heemels, J.M. Schumacher, Dynamical analysis of linear passive networks with ideal diodes, Part II: consistency of a time-stepping method, Technical Report 00 I/03, Eindhoven University of Technology, Department of Electrical Engineering, Measurement and Control Systems, Eindhoven, The Netherlands, 2000.

[4] R.W. Cottle, J.-S. Pang, R.E. Stone, The Linear Complementarity Problem, Academic Press, Boston, 1992.

[5] P. Dupuis, Large deviations analysis of reflected diffusions and constrained stochastic approximation algorithms in convex sets, Stochastics 21 (1987) 63-96.

[6] P. Dupuis, A. Nagurney, Dynamical systems and variational inequalities, Ann. Oper. Res. 44 (1993) 9-42.

[7] A.F. Filippov, Differential equations with discontinuous right-hand side, Mat. Sb 51 (1960) 99-128 (in Russian) (English translation: Am. Math. Soc. Transl. 62 (1964)).

[8] P.T. Harker, J.-S. Pang, Finite-dimensional variational inequality and nonlinear complementarity problems: a survey of theory, algorithms and applications, Math. Programming Ser. B 48 (1990) 161-220.

[9] W.P.M.H. Heemels, Linear complementarity systems: a study in hybrid dynamics, Ph.D. Dissertation, Eindhoven University of Technology, Department of Electrical Engineering, Eindhoven, The Netherlands, 1999.

[10] W.P.M.H. Heemels, M.K. Çamlibel, J.M. Schumacher, Dynamical analysis of linear passive networks with ideal diodes, Part I: well-posedness, Technical Report 00 I/02, Eindhoven University of Technology, Dept. of Electrical Engineering, Measurement and Control Systems, Eindhoven, The Netherlands, 2000.

[11] W.P.M.H. Heemels, J.M. Schumacher, S. Weiland, Applications of complementarity systems, Proceedings of the European Control Conference, Karlsruhe, Germany, 1999.

[12] W.P.M.H. Heemels, J.M. Schumacher, S. Weiland, The rational complementarity problem, Linear Algebra Appl. 294 (1-3) (1999) 93-135.

[13] W.P.M.H. Heemels, J.M. Schumacher, S. Weiland, Linear complementarity systems, SIAM J. Appl. Math. 60 (4) (2000) $1234-1269$. 
[14] Y.J. Lootsma, A.J. van der Schaft, M.K. Çamlibel, Uniqueness of solutions of relay systems, Automatica 35 (3) (1999) 467-478.

[15] P. Lötstedt, Mechanical systems of rigid bodies subject to unilateral constraints, SIAM J. Appl. Math. 42 (1982) 281-296.

[16] D.G. Luenberger, Optimization by Vector Space Methods, Wiley, Chichester, 1969.

[17] J.J. Moreau, Décomposition orthogonale d'un espace hilbertien selon deux cônes mutuellement pôlaires, C.R. Acad. Sci. Paris 255 (1962) 238-240.

[18] A. Nagurney, D. Zhang, Projected Dynamical Systems and Variational Inequalities with Applications, Kluwer, Dordrecht, 1996

[19] F. Pfeiffer, C. Glocker, Multibody Dynamics with Unilateral Contacts, Wiley, Chichester, 1996.

[20] A. Pnueli, J. Sifakis (Guest Eds.), Special issue on hybrid systems, Theoret. Comput. Sci. 138 (1995)

[21] R.T. Rockafellar, Convex Analysis, Princeton University Press, Princeton, NJ, 1970.
[22] W. Rudin, Principles of Mathematical Analysis, McGraw-Hill, New York, 1976.

[23] A.J. van der Schaft, System Theoretic Descriptions of Physical Systems, CWI Tract 3, CWI, Amsterdam, 1984.

[24] A.J. van der Schaft, J.M. Schumacher, The complementaryslackness class of hybrid systems, Math. Control Signals Systems 9 (1996) 266-301.

[25] A.J. van der Schaft, J.M. Schumacher, Complementarity modelling of hybrid systems, IEEE Trans. Automat. Control 43 (1998) 483-490.

[26] A.J. van der Schaft, J.M. Schumacher, An Introduction to Hybrid Dynamical Systems, Lecture Notes in Control and Information Sciences, Vol. 251, Springer, London, 2000.

[27] A. Seeger, Eigenvalue analysis of equilibrium processes defined by linear complementarity conditions, Linear Algebra Appl. 292 (1999) 1-14.

[28] A.V. Skorokhod, Stochastic equations for diffusions in a bounded region, Theory Probab. Appl. 6 (1961) 264-274. 